\title{
An axiomatic approach to the design and operation of a wood pellet production line
}

\author{
Fábio Rodrigues ${ }^{1}$, João Fradinho ${ }^{2 *}$, Miguel Cavique ${ }^{3}$, António Gabriel-Santos ${ }^{2}$ and António Mourão ${ }^{2}$ \\ ${ }^{1}$ DEMI, Faculdade de Ciências e Tecnologia, Universidade NOVA de Lisboa, Campus de Caparica, 2829-516 Caparica, Portugal \\ ${ }^{2}$ UNIDEMI \& DEMI, Faculdade de Ciências e Tecnologia, Universidade NOVA de Lisboa, Campus de Caparica, 2829-516 Caparica, \\ Portugal \\ ${ }^{3}$ UNIDEMI \& CINAV, Escola Naval, Base Naval de Lisboa - Alfeite, 2810-001 Almada, Portugal
}

\begin{abstract}
With the recent search for sustainable energies, the use of forest residues has never been so important and profitable. Biomass production companies are in a constant search for new methods to reuse what is otherwise seen as waste. In this context, wooden pellets have been a booming market. In Portugal, its production increased from 400000 to 1200000 metric tons per year in the period 2008-2014. This production represents a growth of $300 \%$ in 6 years, most of it used for exportation. The manufacturing process is empiric and lacks a scientific approach. In this paper, Axiomatic Design is used to analyse a production line of wooden pellets aiming to identify couplings, both in the equipment and in the production process, and to propose solutions that follow the independence axiom.
\end{abstract}

\section{Introduction}

Axiomatic Design (AD) is a rational framework that leads and guides designers along the design phases [1]. It is based on logical principles where designers can support their decisions, instead of the traditional form based on empiricism, intuition, and trial-and-error. "The ultimate goal of axiomatic design is to establish a scientific basis for design and to improve design activities by providing the designer with a theoretical foundation based on logical and rational thought processes and tools" [2]. The AD key-elements are the design domains, the mapping and decomposition process, the design axioms and the design matrix [2, 3, 4]. They provide a model of the design process, a way to organize the requirements and criteria for "good" designs. Axiomatic Design offers guidance for the creation of good designs. A prominent concept of AD is the concept of independence. It points that good designs always have independence between functional requirements (FRs), not only when they are defined, but also after the consequent definition of the design parameters (DPs). In the Axiomatic Design context, independence between FRs marks the difference between "good" and "discouraged" design solutions.

Independence exists when one and one only different DP adjust its FRs. This can be achieved by two forms: or the order by which FRs are adjusted is irrelevant (the design matrix is diagonal), or FRs must be adjusted by a certain order (the design matrix is triangular, lower or upper). In the first case, changes to a DP will only affect its FR and no others (uncoupled design), and in the second case, changes to a DP will only affect its FR and all the ones lower or upper (decoupled design). In all other situations, changes to a DP will affect its FR and other FRs in the system (coupled design). As per the Axiomatic Design viewpoint, uncoupled, decoupled and coupled designs are respectively the most preferred, acceptable and discouraged. Under this framework, independence is a characteristic that the designer always must seek during the design process. To do so, several strategies are possible to follow, namely:

- Increase FRs tolerances;

- Select a different set of DPs

- Redefine Customer Needs (CN) or Constraints (C) with the stakeholders;

- Optimize the design;

- Add some DPs in order to achieve independence for some FRs [5].

Although not always possible, coupling must be avoided as it means complexity. In other words, independence is a permanent goal in the $\mathrm{AD}$ context.

This paper proposes to analyse a wood pellet current industrial process under the Axiomatic Design perspective. The production line will be examined at the light of the Independence Axiom. New design solutions will be proposed whenever the lack of independence is verified. The ultimate goal is to identify and propose a better production process.

With the recent search for sustainable energies, the use of forest residues has never been so important. Biomass production companies are in a constant search for new ways, such as wooden pellets, to reuse what is otherwise seen as waste. This type of industrial facility has been developed based on ad hoc improvements to industrial systems that exist for several decades. This is the first time that this type of industry has been rationally analysed based on Axiom Design.

\footnotetext{
Corresponding author: jmf@,fct.unl.pt
} 


\section{Characterization of the wood pellet production line}

Figure 1 presents a flowchart of the wood pellet production process.

The wooden pellet production process is based on two main raw materials: woodchips (fragments with 30 to $100 \mathrm{~mm}$ of length) and sawdust (5 to $15 \mathrm{~mm}$ ). These materials are mixed and stored in piles outside the factory. Before entering the process these materials must be dimensionally separated through a $15 \mathrm{~mm}$ mesh rotary sieve. The woodchips over $15 \mathrm{~mm}$ will be ground in a hammer mill with a $15 \mathrm{~mm}$ mesh. The output of the mill will join the chips up to $15 \mathrm{~mm}$ from the sieve resulting in a homogeneous sawdust mix. The rotary screen is used with the aim of optimizing the production of the hammer mill.

The sawdust still has a high moisture content (around $50 \%$ ). To produce quality pellets, it is empirically known that materials should have a moisture content of about $12 \%$, so the next production step is the drying.

The drying of the sawdust is made through a rotational drum dryer. With approximately $2 \mathrm{~m}$ diameter and $7 \mathrm{~m}$ length, the rotating drum carries the sawdust within, ensuring a homogeneous drying of the material. Drying occurs as a result of the hot airflow that is forced in co-current through the dryer. The final material is a homogeneous sawdust mix with a moisture content of about $12 \%$.

At this point, the material is not yet ready for pelletizing because, to have good agglomeration characteristics, it is necessary to reduce dimensions to around $4 \mathrm{~mm}$. The reason why this is not done in the first milling is that the moisture content of the raw materials makes it much more difficult to mill. On the other hand, drying is much more efficient when working with low dimensions of fragments. Therefore, it is preferable to mill the materials on two occasions: before the drying to make it more efficient, and after the drying to reach the desired dimensions.

After obtaining a mix of sawdust with around $4 \mathrm{~mm}$ and moisture content below $12 \%$, the material starts the pelletizing process. In this production step, the material enters a pelletizing machine that forces the mix through a matrix. The matrix has holes with around 6 to $8 \mathrm{~mm}$ and the pressure makes the material agglomerate, this process produces heat, that causes the material to form a type of coating, completely natural (lignin), that will ensure that the pellets maintain the geometry that is given by the matrix. After passing through the matrix, the pellets are cut.

The pelletizing process generates a lot of heat, and the pellets coming from the pelletizer have high temperatures, so it's important to cool them before they advance further into the production line. This cooling is not only important for the mechanisms that will work with the pellets, but also removes any surplus moisture content.

In this stage, the pellets have lots of small particles attached. These particles are caused by crushed pellets or are simple sawdust, which mass is much smaller than the production mass. The particles must be removed so that the pellets can be bagged and shipped to the customer. For this reason, in this stage of production, a vibrating sieve removes almost all the particles. The particles are then carried to the second milling again, to reutilize the material and avoid waste.

Finally, the pellets, once cooled and sieved, are bagged. The bagging is done using a scale that weighs the pellets necessary to fill the bag and then transfers into it. The bags can be of the domestic kind $(15 \mathrm{~kg})$ or big bags $(1000 \mathrm{~kg})$ typically used for industrial clients.

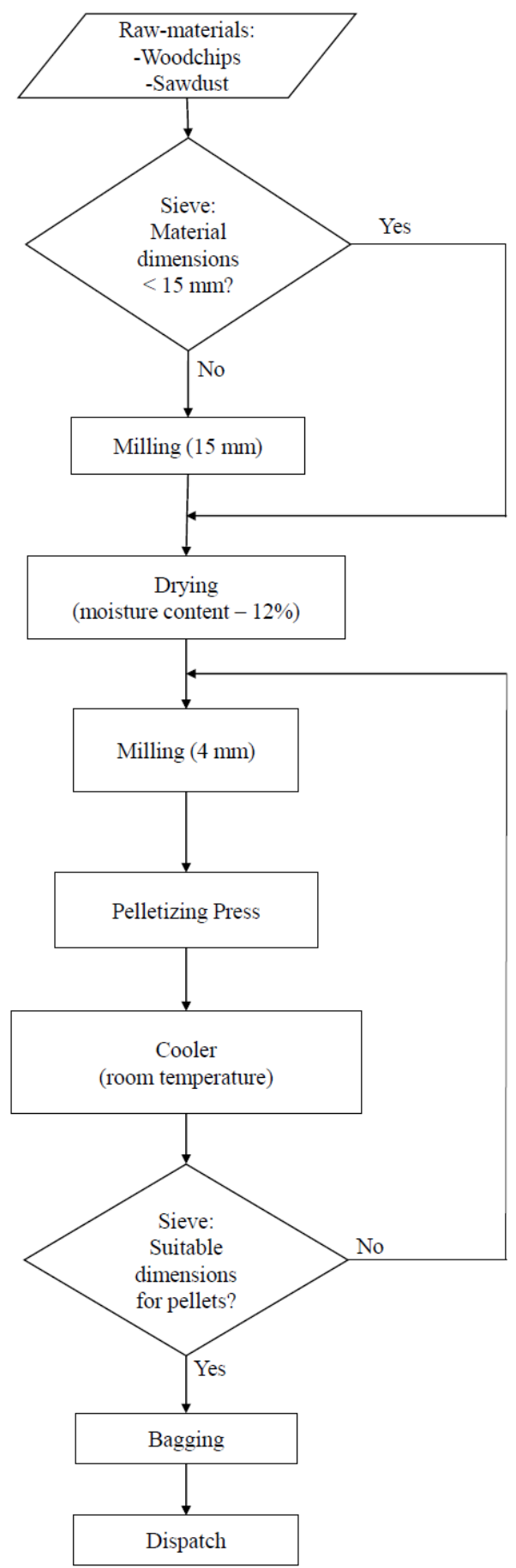

Fig. 1. Flowchart of the wood pellet process. 


\section{An axiomatic approach}

The purpose of the production line is the transformation of the raw materials, woodchips (Figure 2) and sawdust (Figure 3), into wooden pellets (Figure 4).

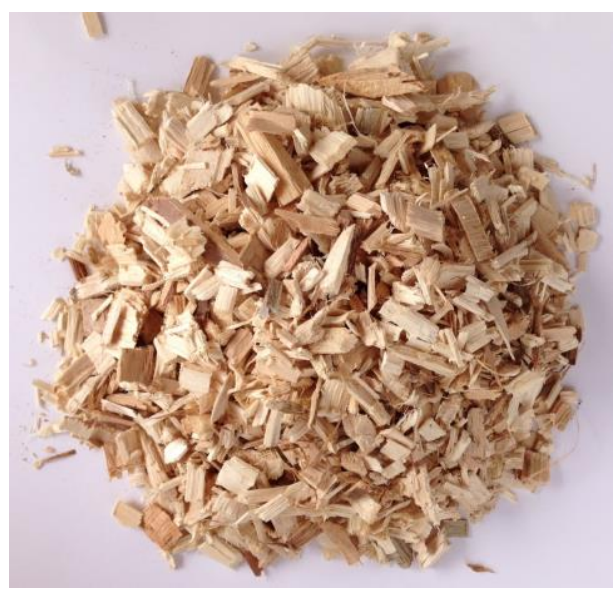

Fig. 2. Woodchips

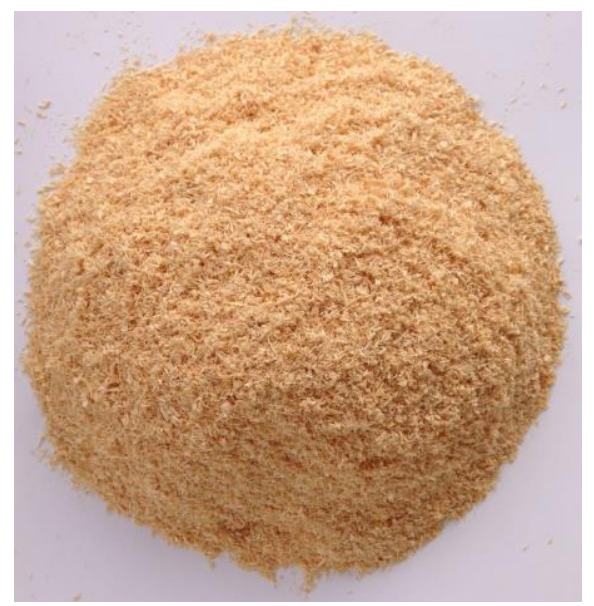

Fig. 3. Sawdust.

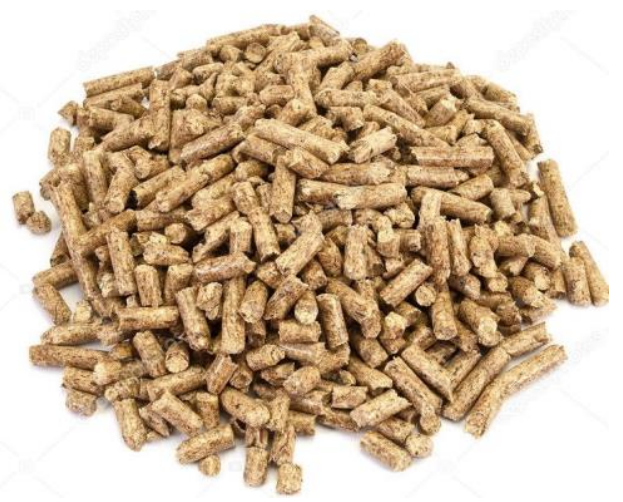

Fig. 4. Wooden pellets.

The line was analysed under an Axiomatic Design viewpoint. The mapping between the functional and the physical domains was done on three levels. In the following text some DPs are illustrated with pictures, which are denoted in the different lists of DPs.

On the first level, one may consider

$\mathrm{FR}_{0}$ - Produce wooden pellets
$\mathrm{DP}_{0}-$ Wood pellet production line

which represents the studied case at the highest level.

The decomposition in the two domains was done in zigzag and six functional requirements on the second level were identified. After the observation and study of the line, there were recognized the respective design parameters. Both, FRs and DPs at the second level, are the following:

$\mathrm{FR}_{1}$ - Prepare raw material for pelletization;

$\mathrm{FR}_{2}$ - Pelletize the material;

$\mathrm{FR}_{3}$ - Prepare pellets for delivery;

$\mathrm{FR}_{4}$ - Carry the material between operations;

$\mathrm{FR}_{5}$ - Filter exhaust gases;

$\mathrm{FR}_{6}-$ Prevent the occurrence of fires.

$\mathrm{DP}_{1}$ - Raw material preparation system;

$\mathrm{DP}_{2}$ - Pelletization mechanism;

$\mathrm{DP}_{3}$ - Post-production system;

$\mathrm{DP}_{4}-$ Distribution and feeding system;

$\mathrm{DP}_{5}-$ Filtration and reuse system;

$\mathrm{DP}_{6}-$ Fire detection and extinguishing system.

At this level, the design equation is represented by expression (1).

$$
\left\{\begin{array}{l}
F R_{1} \\
F R_{2} \\
F R_{3} \\
F R_{4} \\
F R_{5} \\
F R_{6}
\end{array}\right\}=\left[\begin{array}{cccccc}
\times & 0 & 0 & 0 & 0 & 0 \\
0 & \times & 0 & 0 & 0 & 0 \\
0 & 0 & \times & 0 & 0 & 0 \\
0 & 0 & 0 & \times & 0 & 0 \\
0 & 0 & 0 & 0 & \times & 0 \\
0 & 0 & 0 & 0 & 0 & \times
\end{array}\right]\left\{\begin{array}{l}
D P_{1} \\
D P_{2} \\
D P_{3} \\
D P_{4} \\
D P_{5} \\
D P_{6}
\end{array}\right\}
$$

The functional domain is composed of three process requirements (pre-production, production, and postproduction) and three general support systems.

The type of design matrix evidences an uncoupled design because the mass of material coming back (Figure 1) is neglectable compared to the production. Therefore, non-diagonal elements can be ignored. The independence of functional requirements was expected at such a high level due to the autonomy of production. This does not mean that the whole process is uncoupled, and couplings are expected as the decomposition process progresses to more detailed levels.

In the next phase of decomposition (third level) each FR of the second level will be decomposed in several FRs that will have their DPs.

\subsection{Decomposition of $F_{R_{1}}$ - Prepare raw material for pelletization}

The woodchips fragments arrive at the company with dimensions up to $150 \mathrm{~mm}$ and are stored in outdoor piles where it often reaches humidity levels of about $50 \%$.

The pelletization operation requires raw materials with a moisture content of less than $12 \%$ and dimensions less than $4 \mathrm{~mm}$.

The large reduction range implies that grinding is done in two phases: the first one up to $15 \mathrm{~mm}$ and the second one up to $4 \mathrm{~mm}$. Drying is more effective with reduced size fragments but also causes dimensional 
variations. Thus to have better dimensional control, the drying operation is performed between the two grinding operations.

The first device of the line is a sieve to withdraw fragments of less than $15 \mathrm{~mm}$ that do not need to pass through the mill, which has a $15 \mathrm{~mm}$ mesh.

Thus, in the preparation of raw materials, four functional requirements emerge:

$\mathrm{FR}_{11}$ - Separate fragments up to $15 \mathrm{~mm}$;

$\mathrm{FR}_{12}$ - Reduce dimensions to less than $15 \mathrm{~mm}$;

$\mathrm{FR}_{13}$ - Reduce moisture content to less than $12 \%$;

$\mathrm{FR}_{14}$ - Regularize dimensions to less than $4 \mathrm{~mm}$.

The answer to how these four functions are achieved is a set of four machines that represent the DPs:

$\mathrm{DP}_{11}$ - Sieve (15 mm mesh);

$\mathrm{DP}_{12}$ - Hammer mill (15 mm mesh);

$\mathrm{DP}_{13}$ - Drum dryer (Figure 5);

$\mathrm{DP}_{14}$ - Hammer mill (4 mm mesh) (Figure 6).

Expression (2) represents the resulting design equation.

$$
\left\{\begin{array}{l}
F R_{11} \\
F R_{12} \\
F R_{13} \\
F R_{14}
\end{array}\right\}=\left[\begin{array}{cccc}
\times & 0 & 0 & 0 \\
0 & \times & 0 & 0 \\
0 & 0 & \times & 0 \\
0 & 0 & 0 & \times
\end{array}\right]\left\{\begin{array}{l}
D P_{11} \\
D P_{12} \\
D P_{13} \\
D P_{14}
\end{array}\right\}
$$

Therefore, the design of raw material preparation is uncoupled.

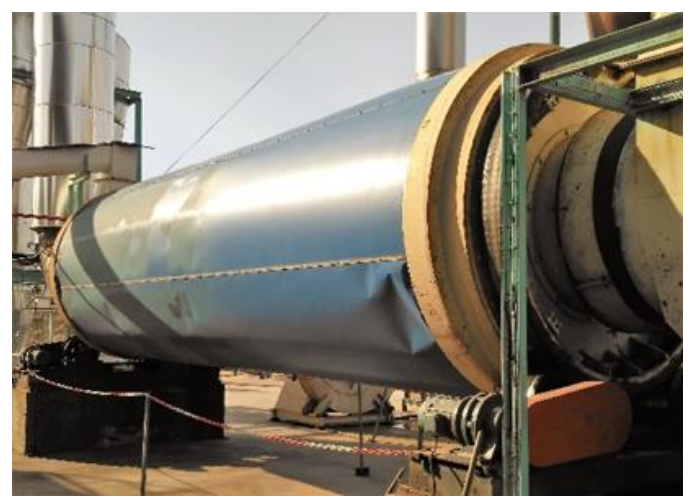

Fig. 5. Drum dryer.

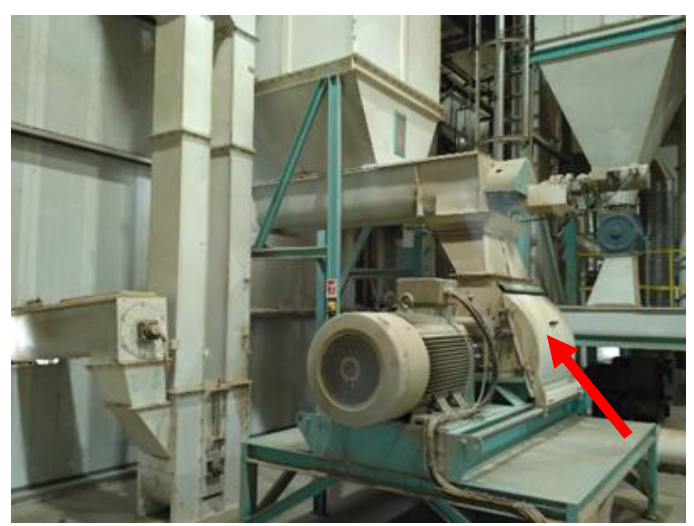

Fig. 6. Hammer mill.

\subsection{Decomposition of $\mathrm{FR}_{\mathbf{2}}$ - Pelletize the material}

The main equipment of the production line is the pelletizing press. Here the woodchip fragments are agglomerated by pressure and forced to pass through a matrix, which will give the cylindrical geometry with a certain diameter and length.

Pellets consistency is due to the pressure the press rollers exert on the mix forcing the material to pass through the matrix. It generates heat and causes the migration of the natural wood binder.

Due to the high pressures inside the press, there are substantial frictional forces between the material and the die. As lubricants cannot be used to avoid contamination, water vapor is used locally to reduce friction.

In this operation, the functional requirements are:

$\mathrm{FR}_{21}$ - Give pellets diameters 6 to $8 \mathrm{~mm}$;

$\mathrm{FR}_{22}$ - Give pellets a length 3.15 to $40 \mathrm{~mm}$;

$\mathrm{FR}_{23}$ - Give the pellets the necessary density;

$\mathrm{FR}_{24}$ - Reduce the frictional forces between

material and the matrix.

These requirements are achieved through the following physical systems:

$\mathrm{DP}_{21}$ - Circular matrix (Figure 7);

$\mathrm{DP}_{22}$ - Cutting comb;

$\mathrm{DP}_{23}$ - Press rollers;

$\mathrm{DP}_{24}$ - Water vapor jet.

Equation (3) shows the design equation demonstrating that this is a coupled design:

$$
\left\{\begin{array}{l}
F R_{21} \\
F R_{22} \\
F R_{23} \\
F R_{24}
\end{array}\right\}=\left[\begin{array}{llll}
\times & 0 & 0 & 0 \\
0 & \times & 0 & 0 \\
0 & 0 & \times & \times \\
0 & 0 & \times & \times
\end{array}\right]\left\{\begin{array}{l}
D P_{21} \\
D P_{22} \\
D P_{23} \\
D P_{24}
\end{array}\right\}
$$

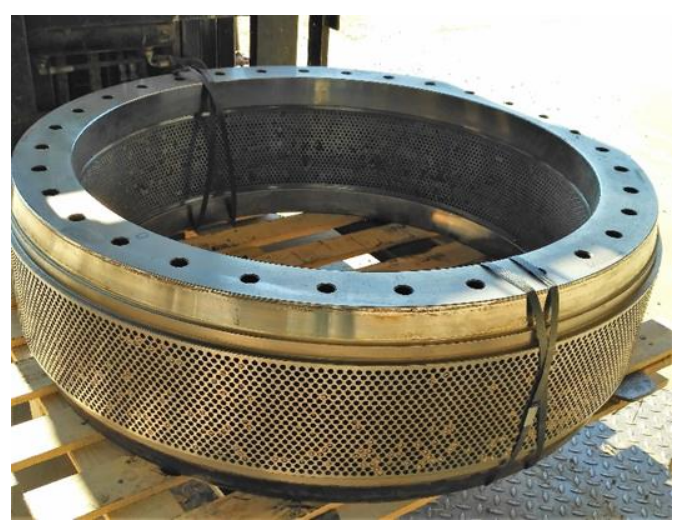

Fig. 7. Circular matrix.

In this operation, there is a coupling because the water vapor jet also affects the pellets density and the press rollers contribute to the frictional forces.

This situation could be improved by the introduction of a control system in the water vapor jets. The right amount of water vapor would be automatically added as a function of the moisture level read in the entrance of the press. With this new system, the density of the pellets would not be affected. Thus, the proposed new DP is:

$D P^{\prime}{ }_{24}$ - Water vapor automatic system. 
The new design equation (4) would then represent a decoupled design.

$$
\left\{\begin{array}{l}
F R_{21} \\
F R_{22} \\
F R_{23} \\
F R_{24}
\end{array}\right\}=\left[\begin{array}{cccc}
\times & 0 & 0 & 0 \\
0 & \times & 0 & 0 \\
0 & 0 & \times & 0 \\
0 & 0 & \times & \times
\end{array}\right]\left\{\begin{array}{c}
D P_{21} \\
D P_{22} \\
D P_{23} \\
D P_{24}^{\prime}
\end{array}\right\}
$$

\subsection{Decomposition of $\mathrm{FR}_{\mathbf{3}}$ - Prepare pellets for delivery}

After the press, pellets are at a very high temperature and also contain small particles known as "fines".

In order to have an appropriate storage process, one has to meet the following requirements:

$\mathrm{FR}_{31}$ - Cool the pellets;

$\mathrm{FR}_{32}$ - Remove fines;

$\mathrm{FR}_{33}$ - Pack the pellets.

The DPs for these functions are the following:

$\mathrm{DP}_{31}$ - Cooler;

$\mathrm{DP}_{32}$ - Vibrating screen;

$\mathrm{DP}_{33}$ - Pellet bagging machine (Figure 8).

The post-production phase has the design equation presented in expression (5), which represents an uncoupled design.

$$
\left\{\begin{array}{l}
F R_{31} \\
F R_{32} \\
F R_{33}
\end{array}\right\}=\left[\begin{array}{lll}
\times & 0 & 0 \\
0 & \times & 0 \\
0 & 0 & \times
\end{array}\right]\left\{\begin{array}{l}
D P_{31} \\
D P_{32} \\
D P_{33}
\end{array}\right\}
$$

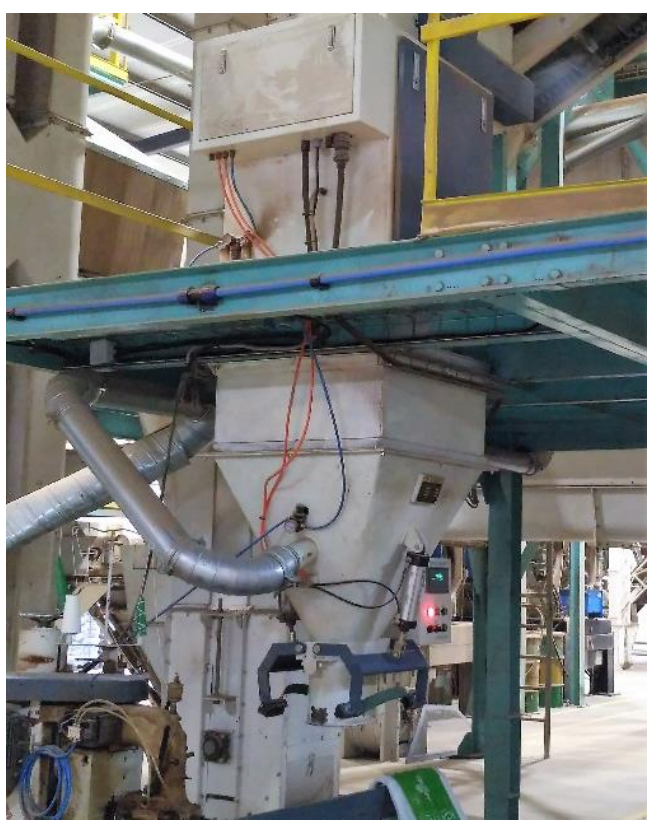

Fig. 8. Pellet bagging machine.

\subsection{Decomposition of $\mathrm{FR}_{\mathbf{4}}$ - Carry the material between operations}

Throughout the production line, conveyors and silos allow uncoupling the design, according to theorems 24 and 25. The FRs are:

$\mathrm{FR}_{41}$ - Store woodchip;
$\mathrm{FR}_{42}$ - Convey woodchips (40-150 mm - 50\% moisture).

This woodchip is later crushed with dimensions up to $15 \mathrm{~mm}$ (sawdust).

$\mathrm{FR}_{43}$ - Store moist sawdust;

$\mathrm{FR}_{44}$ - Convey moist sawdust (up to $15 \mathrm{~mm}-50 \%$ moisture).

The sawdust is then dried reducing its moisture content by up to $12 \%$.

$\mathrm{FR}_{45}$ - Store dried sawdust;

$\mathrm{FR}_{46}$ - Convey dried sawdust (up to $15 \mathrm{~mm}-12 \%$ moisture).

The sawdust is then crushed again to reach the dimensions required for the production of pellets $(4 \mathrm{~mm})$.

$\mathrm{FR}_{47}$ - Store final raw material;

$\mathrm{FR}_{48}$ - Convey processed material (up to $4 \mathrm{~mm}$ $12 \%$ moisture).

This processed material is ready to be transformed into pellets and send to the pelletizing press. After the press, the pellets must be conveyed and stored.

$\mathrm{FR}_{49}$ - Store pellets;

$\mathrm{FR}_{410}$ - Convey pellets.

There are several types of materials to store and transport. The equipment used in the process must have into account the different properties of the materials to be transported.

The corresponding DPs are:

$\mathrm{DP}_{41}$ - Woodchip silo;

$\mathrm{DP}_{42}$ - Belt conveyor;

$\mathrm{DP}_{43}$ - Sawdust silo;

$\mathrm{DP}_{44}$ - Pneumatic conveyor;

$\mathrm{DP}_{45}$ - Sawdust silo;

$\mathrm{DP}_{46}$ - Worm conveyor 1;

$\mathrm{DP}_{47}-\mathrm{Silo}$

$\mathrm{DP}_{48}$ - Worm conveyor 2;

$\mathrm{DP}_{49}$ - Pellet silo (Figure 9);

$\mathrm{DP}_{410}$ - Belt conveyor.

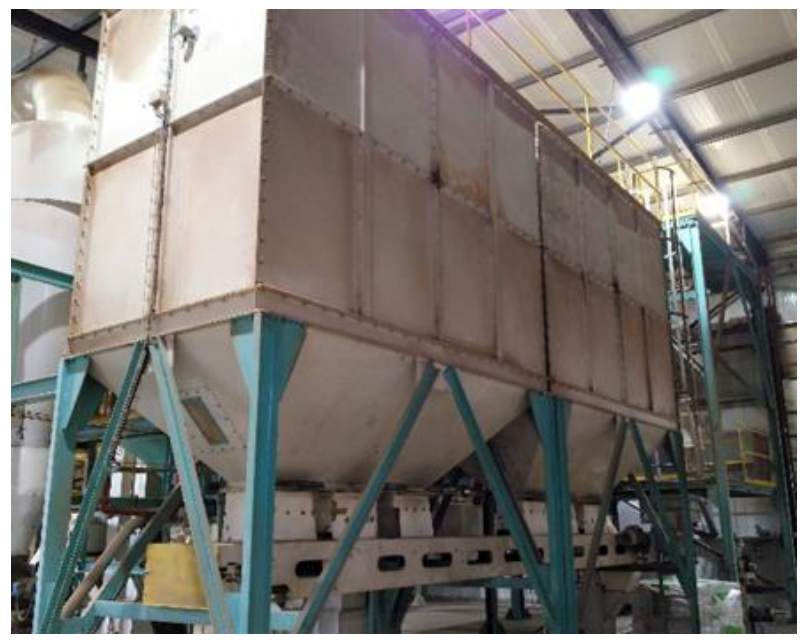

Fig. 9. Pellet silo.

\subsection{Decomposition of $\mathrm{FR}_{\mathbf{5}}$ - Filter exhaust gases}

The processes that are used for the preparation of the raw material and for the production of the pellets produce particles of small dimension. These particles cannot be 
released into the atmosphere, and its reuse is very beneficial.

The two hammer mills are the main dust generators. Since the material at its outlet is pneumatically transported, it is crucial that the air is filtered.

Drying is another process, in which the exhaust gas must be controlled. The hot air in the dryer is produced by the combustion of wood and cork dust and is directly in contact with the material to be dried. Then, the exhaust gas is saturated with wood particles and ashes. Therefore, its exhaustion to the atmosphere would be quite harmful, so that it must be passed through a gas cleaning system.

Thus, two functional requirements are defined as:

$\mathrm{FR}_{51}$ - Filter the air at the outlet of the mills;

$\mathrm{FR}_{52}$ - Filter the air at the dryer outlet.

These functions are ensured by the following systems:

$\mathrm{DP}_{51}-$ Air filtering system (mills) (Figure 10);

$\mathrm{DP}_{52}$ - Gas cleaning and filtering station.

In this gas filtration operation, the design matrix is an uncoupled design.

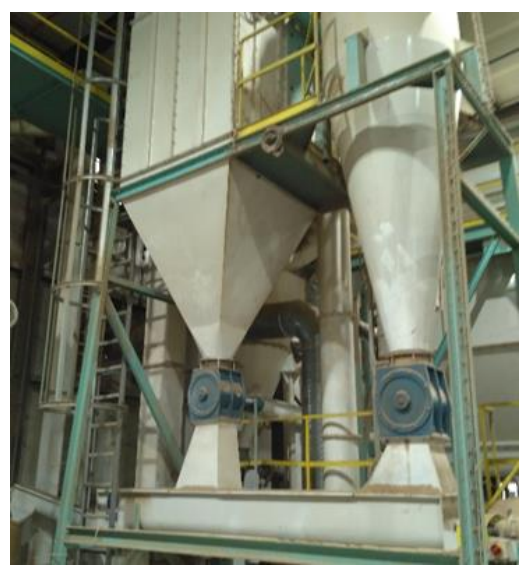

Fig. 10. Air filtering system.

\subsection{Decomposition of $\mathrm{FR}_{6}$ - Prevent the occurrence of fires}

This line processes dust and woodchips that are excellent fuels. Additionally, dust particles have explosive characteristics due to the large surface per unit volume. It is, therefore, necessary to have a system that detects and extinguishes any occurrence of fire.

The first stage in this system must be the detection of sparks or abnormal temperature increases that may occur in the transport of material.

After the detection of the initial stages of a fire, it is imperative to proceed with immediate extinction. Finally, the wet material must be removed from the normal course of the raw material, either to clear the way for regularization of production and to prevent combustion that may not have been extinguished.

Thus, the FRs are defined as:

$\mathrm{FR}_{61}$ - Detect sparks;

$\mathrm{FR}_{62}$ - Moisten affected material;

$\mathrm{FR}_{63}$ - Remove the material from the line.
The installed system has the following design parameters:

$$
\begin{aligned}
& \mathrm{DP}_{61} \text { - Spark and temperature sensor; } \\
& \mathrm{DP}_{62} \text { - Sprinklers; } \\
& \mathrm{DP}_{63} \text { - Material removal system. }
\end{aligned}
$$

This fire design is also an uncoupled design.

Due to the lack of space, the matrix of the second level of decomposition is not shown. Using $D P^{\prime}{ }_{24}$ the design on the second level of decomposition is a decoupled design.

\section{Conclusions}

In this paper, a wood pellet production line was studied. Its main objectives were to analyse it top-down identifying in each of the three levels considered, their functional requirements and their design parameters. It was intended to assess how the independence axiom, that is a fundamental pillar of Axiomatic Design, is being followed.

It was found that the design of the plant relies heavily on the concept of functional independence even without prior knowledge of the Axiomatic Design concepts.

In the decomposition between the functional and physical domains, an FR was identified at the first level and six FRs were identified at the second level. At this level, the project is represented by a diagonal matrix revealing full independence. At the third level, each one of these six FRs was decomposed and gave rise to five uncoupled sub designs and one coupled sub design. In this coupled sub design, the replacement of a DP was proposed in order to have a decoupled design.

The high compliance with the independence axiom is indicative of the good implementation of the production process that makes the company very attractive regarding productivity. Because of the low level of detail to which this study came, any inference about the quality of the design is premature.

The authors gratefully thank the sponsorship of Fundação para a Ciência e Tecnologia through the Strategic Project UID/EMS/00667/2019 - UNIDEMI.

\section{References}

1. G.-J. Park, Analytic Methods for Design Practice. London: Springer-Verlag, (2007)

2. N. P. Suh, Axiomatic Design: Advances and Applications. New York: Oxford University Press, (2001)

3. N. P. Suh, The principles of design. New York: Oxford University Press, (1990)

4. N. P. Suh, Complexity - Theory and Applications. New York: Oxford University Press, (2005)

5. A. M. Gonçalves-Coelho, G. Neştian, M. Cavique, A. Mourão, INT J PRECIS ENG MAN 13, 10. 1837-1843 (2012) 